\title{
Fuzzy Matter-element Safety Assessment of military ATC System Based on Combination Weighting
}

\author{
Ying Liu ${ }^{1, a}$, Xusheng Gan ${ }^{2, b}$, Nan Yang ${ }^{2, c}$ \\ ${ }^{1}$ Xijing College, Xi’an, Shaanxi, 710123, China \\ ${ }^{2}$ Air Traffic Control and Navigation College, Air Force Engineering, University, Xi’an, Shaanxi, 710051, \\ China \\ a liuying111@163.com, b ganxusheng123@163.com, cyangn001@126.com
}

Keywords: Military ATC; Fuzzy Matter-element; AHP; Entropy; Safety Assessment

\begin{abstract}
To improve the safety operation level of military ATC system, according to the fuzzy matter-element analysis theory, a safety assessment method based on combination weighting is proposed on the basis of safety assessment index system of military ATC system. In the method, the weight calculated by Analytic Hierarchy Process and Entropy method respectively are combined to conduct the combination weighting for assessment indices. The example analysis shows that the method has many advantages such as simple step, scientific and reasonable process, consideration of the subjective and objective influence, and also is feasible and effective.
\end{abstract}

\section{Introduction}

With the development of aviation transportation industry, Air Traffic Control (ATC) system operation safety has been increased the complexity and uncertainty. This has undoubtedly increased the difficulty of scientific assessment of ATC system operation safety. Therefore, it is of great practical significance to use the scientific method to comprehensively evaluate the safety of ATC system, which can provide theoretical and technical support for management and decision-making.

Pasquini et al studied the safety assessment method of ATC, and proposed the ATC operation safety application program based on computer technology [1]. Du et al introduced the triangular fuzzy number into Network Analytic Hierarchy Process (ANP) Method, and established the fuzzy network analysis assessment model of ATC system safety [2]. Zhao et al integrated Analytic Hierarchy Process with Entropy Method to build a fuzzy combination safety assessment model for ATC system operation on the basis of aircraft operational data [3].

In view of this, based on fuzzy matter-element analysis, information entropy theory and AHP, the application of fuzzy matter-element analysis method is explored in ATC safety assessment, and its effectiveness is verified by examples.

\section{Safety assessment index system for military ATC system}

Combined with the work content of the military ATC, a diagram of human-machine-environment relationship for airport flight ATC area operation shown in Fig. 1 can be obtained. As can be seen from the figure, the controller of the airport control area is extremely important in the military ATC. He need obtain the information from different channels such as other related ATC departments, civil ATC departments, related artillery units, related airport units, combat duty detachments and radar displays. After processing, the instructions and information that are conducive to flight safety are transmitted to the related aircraft through communication equipment, which is of great responsibility and the pressure on them is quite large. In this process, every link can have problems, but all aspects also need to be considered [4].

Based on this, to establish a safety assessment index system that reflects the actual operation of the military ATC system, it is necessary to follow the basic principles of science, comprehensiveness and 
hierarchy, namely, the assessment index system is structured as the target level, the criterion level and the index level. Among them, the target layer uses a combination assessment index of ATC system to measure the overall safety level of the system; the criteria layer mainly covers the influencing factors of people, equipment, environment and management, among which the management factors are the factors directly or indirectly affecting human factors, equipment factors and environmental factors and are higher than these factors; the index layer is the further refinement of the criterion layer and the specific expression of the main features of the system. On the basis of extensive research on relevant cases, 43 influencing factors are selected through consulting experts and combining actual operation of military ATC system. Then, using the factor analysis function module of SPSS statistical analysis software, the principal component analysis for historical data is carried out. After maximizing the rotation of the variance, we can find that the cumulative contribution rate of the six principal components, $(1 / P) \sum_{i=1}^{m} \lambda_{i} \geq 85 \%$. In order to make the established assessment index system more reflect the safety state of military ATC system operation, in the principal component matrix after the rotation, The 10 influencing factors with cumulative contribution rate are initially selected to construct the safety assessment index system of the military ATC system, as shown in Figure 2.

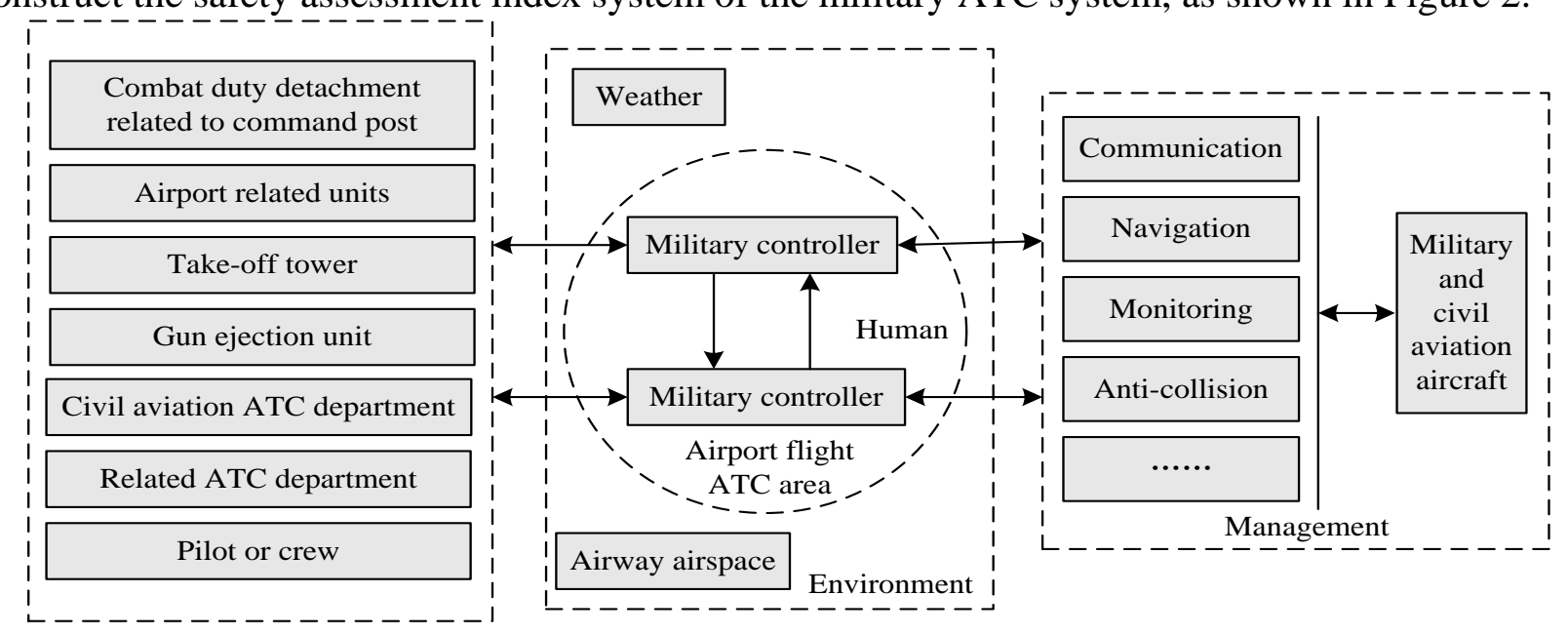

Figure 1 Human-machine-environment relationship of airport flight ATC area operation

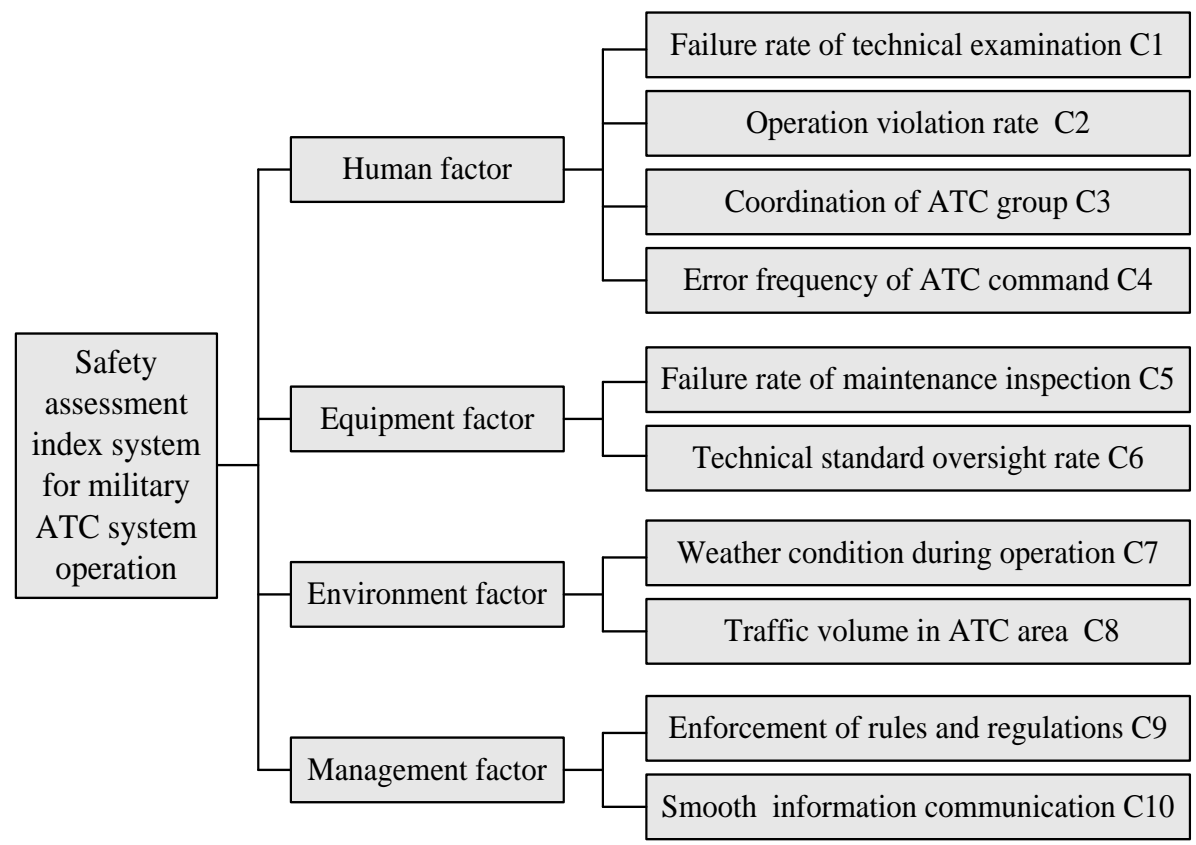

Figure 2 Safety assessment index system of military ATC system operation 


\section{Fuzzy matter-element safety assessment model for military ATC system}

The fuzzy matter-element analysis method [5] was proposed in 1995 by Professor Xiao Fangjun which combines the matter-element analysis theory and the fuzzy set theory to effectively deal with the fuzzy incompatibility problem in the assessment process. And because of its excellent performance, it is widely used in safety assessment of ecology, geology, water and construction. In this paper, a fuzzy matter-element analysis model with combination weighting is applied to the safety assessment of military ATC system. The assessment process is shown in Figure 3.

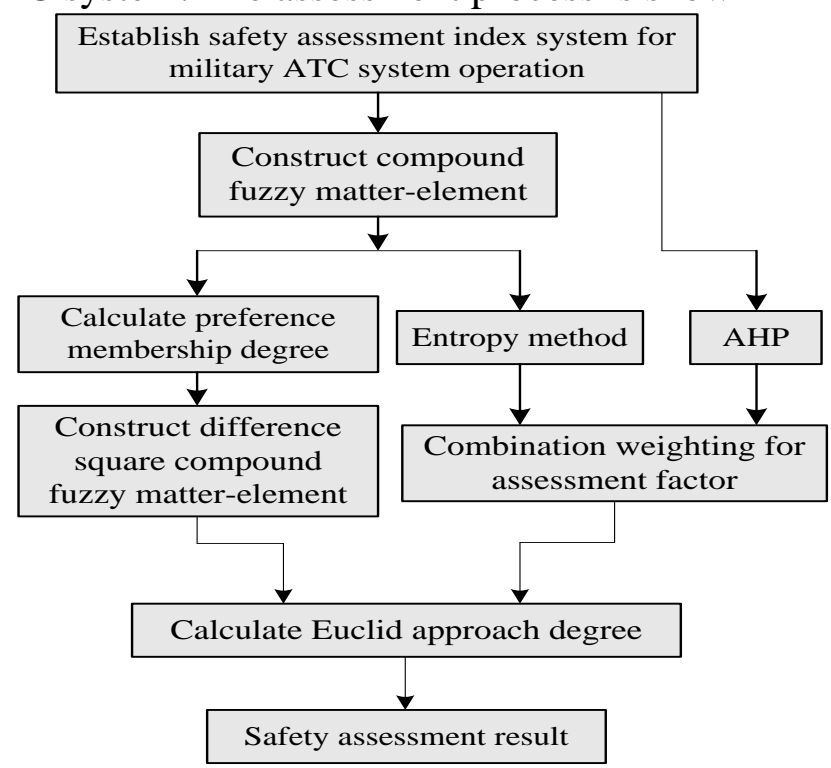

Figure 3 Flow chart of fuzzy matter-element assessment with combination weighting

\section{Combination weighting Based on Entropy method and AHP}

According to the fuzzy matter-element analysis process, to correctly evaluate the operation safety of military ATC system, weighting is a very critical issue. AHP determines the weight according to the analyst's preference for each assessment factor, which is simple and easy to implement, but is subject to subjective factors. The entropy method determines the weight according to the correlation and variation degree of the assessment factor itself, effectively avoiding the influence of human factors, but is vulnerable to interference with random errors in the sample. In the safety assessment of military ATC operation, the paper combines the two and complements each other. On the basis of the principle of minimum discriminant information, we propose to combine the weights obtained by AHP and entropy method to obtain the combined weights, which can strive to achieve the subjective and objective unity for weight determination.

\section{Example analysis}

The overall safety status of 5 ATC areas is evaluated combinationly, and 10 experts in ATC safety were selected to score the assessment index C3, C5, C7, C8 and C10 that could not directly obtain data. The percentage system can be adopted, that is, the scoring range is $[0,100]$, which is invalid beyond this range. Finally, the scores of each index in 5 ATC areas given by the experts and collected are averaged, obtaining raw data is shown in matrix, and thus constitutes a composite fuzzy matter-element. According to actual operation of ATC system, the safety assessment index C1, C2, C4, C6, and C9 are as small as possible; The index C3, C5, C7, C8, and C10 are as large as possible. The calculated values of the two are combined to obtain the preference membership fuzzy matter-element. The combination weights are shown in Table 1.

According to calculating Euclid approach degree, the sorting of safety assessment of 5 ATC areas can be obtained. That is: ATC Area 5> ATC Area 1> ATC Area 4> ATC Area 3> ATC Area 2, of 
which ATC Area 5 is the safest.

$$
R_{5,10}=\left[\begin{array}{ccccc}
0.12 & 0.10 & 0.14 & 0.07 & 0.09 \\
0.04 & 0.02 & 0.05 & 0.01 & 0.02 \\
80 & 78 & 85 & 81 & 92 \\
3 & 7 & 6 & 4 & 5 \\
92 & 90 & 98 & 94 & 95 \\
0.01 & 0.05 & 0.02 & 0.04 & 0.01 \\
85 & 95 & 80 & 90 & 85 \\
75 & 80 & 85 & 80 & 92 \\
0.05 & 0.02 & 0.04 & 0.07 & 0.03 \\
94 & 91 & 92 & 90 & 92
\end{array}\right]^{\mathrm{T}}
$$

Table 1 Combination weights of safety assessment indices for each ATC area

\begin{tabular}{cccc}
\hline Index & AHP Q & $\begin{array}{r}\text { Entropy } \\
\text { method }\end{array}$ & $\begin{array}{c}\text { Comprehensive } \\
\text { weight } w\end{array}$ \\
\hline$C_{1}$ & 0.1221 & 0.0923 & 0.1102 \\
$C_{2}$ & 0.0876 & 0.1075 & 0.0956 \\
$C_{3}$ & 0.1195 & 0.1074 & 0.1147 \\
$C_{4}$ & 0.0793 & 0.0958 & 0.0859 \\
$C_{5}$ & 0.0954 & 0.0902 & 0.0933 \\
$C_{6}$ & 0.1280 & 0.1402 & 0.1329 \\
$C_{7}$ & 0.0971 & 0.0908 & 0.0946 \\
$C_{8}$ & 0.0865 & 0.0922 & 0.0887 \\
$C_{9}$ & 0.1204 & 0.0956 & 0.1104 \\
$C_{10}$ & 0.0641 & 0.0879 & 0.0737 \\
\hline
\end{tabular}

\section{Conclusions}

The operation of military ATC system is characterized by fuzzy, complexity and diversity of indices. The fuzzy matter-element method is used to combinationly evaluate its safety. In the method, it is crucial to weight the indices. For this, a combination weighting method is proposed, that is, AHP is used to calculate the subjective weight, the entropy method is applied to calculate the objective weight, and the principle of minimum discriminant information is introduced to organically combine the two, weighting the indices combinationly. This can achieve the subjective and objective unity of the combination assessment of safety for military ATC.

\section{References}

[1] A. Pasquini, S. Pozzie. Assessment of air traffic management procedures-safety assessment in an experimental environment. Reliability Engineering \& System Safety, 89(1), (2005), 105-117.

[2] H. B. Du, H. Li, L. P. Yuan, X. Li. Risk assessment model for air traffic control based on Fuzzy-ANP method. China Safety Science Journal, 20(12), (2010), 79-85.

[3] Y. F. Zhao, L. Chen, H. Y. Wang. Safety risk assessment of ATM operation based on entropy weight and fuzzy analysis. Aeronautical Computing Technique, 43(4), (2013), 1-5.

[4] P. Stastuy. Risk assessment \& mitigation in ATM. Safety Science, 44(7), (2006), 629-655.

[5] B. Zhang, Q. D. Yong, F. C. Xiao. Fuzzy matter-element analysis. Beijing: Petroleum Industry Press, 1997. 